\title{
Ein Bypass, kein Herzinfarkt
}

VB verfassungsblog.de/ein-bypass-kein-herzinfarkt/

Daniel Thym Di 13 Dez 2011

Di 13 Dez

2011

In den ersten Reaktionen auf das britische Veto des „Fiskalpakts“ war viel von einer „Spaltung“ Europas die Rede. Hinweise auf die rechtlichen Fallstricke eines völkerrechtlichen Ergänzungsvertrags werden ergänzt um Spekulationen über eine Neugründung der EU ohne das Vereinigte Königreich. Ein Blick in das Kleingedruckte des Fiskalpakts zeigt jedoch, dass die rechtlich-institutionelle Revolution ausbleibt. Die Währungsunion mag volkswirtschaftlich im Bestand bedroht sein und auch politisch eine Neuauflage des britischen Widerstandsgeists in den frühen 1990er-Jahren erleben (als die Ratifikation des Maastricht-Vertrags beinahe an einer Revolte der britischen Konservativen scheiterte). Rechtlich verbindet sich der Fiskalpakt jedoch überraschend harmonisch mit dem geltenden Vertragsrecht.

Dies ist kein Zufall. Zu den Ritualen der europäischen Integrationspolitik gehören periodische Diskussionen über die Herausbildung eines Kerneuropa. Speziell in Zeiten der Krise wird regelmäßig eine abgestufte Integration als möglicher Ausweg beschworen, der sodann zumeist ausbleibt. Mit Ausnahme des Schengen-Regimes wurde bislang kein größeres Kerneuropaprojekt verwirklicht. Die wiederholten Debatten bewirkten jedoch die Aufnahme vorsorglicher Regeln für den vertragsinternen Ausbau verschiedener Integrationsgeschwindigkeiten, die bislang ein Schattendasein führten. Eben diese Regeln erkennt der europarechtlich geschulte Leser jedoch bei der Lektüre des Abschlusskommuniqués des Euro-Gipfels. Der künftige „Fiskalpakt“ wird nur sehr begrenzt auf völkerrechtlicher Grundlage verwirklicht werden. Er führt zu keiner Spaltung Europas und auch zu keiner unkontrollierten Abkehr von der supranationalen Integrationsmethode.

\section{Begrenzter „Fiskalpakt“}

In der Berichterstattung über den jüngsten Euro-Gipfel dominiert das britische Veto gegen die Vertragsänderung mit der Folge eines völkerrechtlichen Vertragsschlusses außerhalb des EU-Rahmens. Tatsächlich soll dieser Ergänzungsvertrag nach dem Abschlusskommuniqué jedoch nur diejenigen Gipfelbeschlüsse betreffen, die nicht auf Grund des geltenden Rechts verwirklicht werden können. Dies betrifft im Kern zwei Punkte: erstens die Einführung einer Schuldenbremse mit automatischem Korrekturmechanismus und zweitens umgekehrte Mehrheitsentscheidungen bei Verstößen gegen den Stabilitätspakt. Weitere Projekte, etwa rechtsverbindliche Durchgriffsrechte der EU-Kommission auf das Haushaltsrecht von Defizitsündern, finden sich im Abschlusskommuniqué nicht. Frau Merkel konnte sich hier nicht durchsetzen.

Diese doppelte Neuerung verdient kaum die hochtrabende Bezeichnung als „Fiskalunion“ oder gar „Wirtschaftsregierung“ - und zwar auch dann nicht, wenn man die intergouvernementalen Beschlüsse des früheren Euro-Plus-Pakts hinzunimmt. Eine echte Fiskalunion mit Befugnissen in der Steuer-, Sozial- oder Wirtschaftspolitik, von der Jürgen Habermas träumt, wird der völkerrechtliche Ergänzungsvertrag, der bis März 2012 ausgehandelt werden soll, nicht umfassen. Dies bedeutet nicht, dass der Euro-Gipfel derartige Reformen ausschloss. Erwogen werden Schritte zur Vertiefung der Wirtschaftsunion durchaus - jedoch außerhalb des intergouvernementalen Fiskalpakts.

\section{Vorkehrungen für ein „Kerneuropa“}

Als Wolfgang Schäuble vor 17 Jahren gemeinsam mit Karl Lamers die Bildung eines Kerneuropa vorschlug, führte dies wegen des impliziten Ausschlusses Italiens und anderer Südstaaten von der Währungsunion zu politischen Verwicklungen. Ungeachtet dieses Streits zielte der Vorschlag jedoch auf eine institutionelle Weiterentwicklung der EU durch eine flexible Entscheidungsfindung in Form einer engeren Zusammenarbeit der Euro-Staaten innerhalb des EU-Rahmens. Eben dieses Ziel wurde aufgrund eines deutsch-französischen Vorschlags im Vertrag von Amsterdam erreicht, der nicht nur das Schengener Recht vergemeinschaftete, sondern zugleich einen Mechanismus unter dem sperrigen Titel „verstärkte Zusammenarbeit“ bereitstellte, der 
einigen Mitgliedstaaten die Annahme regulären EU-Rechts im kleinen Kreis ermöglicht.

Nunmehr heißt es im Gipfelkommuniqué: „Wir kommen überein ... konsequenter auf die Verstärkte Zusammenarbeit zurückzugreifen." Dies meint nicht etwa eine engere Kooperation auf völkerrechtlicher Grundlage, sondern die Nutzung des neuen Mechanismus, der mit dem Vertrag von Nizza reformiert wurde und nunmehr in Art. 326-334 AEUV geregelt wird. Die „verstärkte Zusammenarbeit“ begründet alles andere als eine vage Option ohne Rechtsverbindlichkeit. Im Gegenteil: es werden reguläre Richtlinien und Verordnungen mit supranationaler Rechtswirkung angenommen; es gilt das reguläre Gesetzgebungsverfahren unter Beteiligung des Europäischen Parlaments, ohne dass die Rechtsakte eine Ratifikation durch die nationalen Parlamente erforderten.

Offenbar erstreben die Staats- und Regierungschefs auf diesem Wege eine Vertiefung der Wirtschaftsunion, wie dies auch Herman van Rompuy in seinem Bericht zur Vorbereitung des Gipfels vorgeschlagen hatte. Schon bald könnte dies Vorhaben auf die Probe gestellt werden: Wenn unter 27 Mitgliedstaaten die erforderliche Einstimmigkeit für die Annahme der Kommissionsvorschläge für eine Finanzmarkttransaktionssteuer oder auch die konsolidierte Körperschaftsteuer-Bemessungsgrundlage scheitert, können diese Schritte in Richtung Fiskalunion im kleinen Kreis vereinbart werden - ohne dass dem Vereinigten Königreich ein Veto zukäme. Über die Errichtung einer verstärkten Zusammenarbeit entscheidet der Rat nämlich mit qualifizierter Mehrheit (Art. 20 Abs. 2 i.V.m. Art. 16 Abs. 3 EUV).

Der Lissabon-Vertrag gestaltet die verstärkte Zusammenarbeit mit einem weiteren Vorzug aus: Im kleinen Kreis können Mehrheitsentscheidungen über den Inhalt der Rechtsakte vereinbart werden, ohne dass die "outs" dies verhindern können. Hierdurch kann schrittweise eine Annäherung an eine „echte“ Wirtschaftsregierung erfolgen, etwa in der Steuer-, Arbeitsmarkt- und Sozialpolitik. Wenn diese Option nicht genutzt wird, fehlt es am politischen Integrationswillen. Völkerrechtliche Verträge sind hier ebenso unnötig wie das deutsch-französische Insistieren auf eine Vertragsänderung.

\section{Vertragsinterner Ausbau der Haushaltsaufsicht}

Eine zentrale Forderung der Bundesregierung ist die Stärkung des Stabilitätspakts in Form einer präventiven und reaktiven Haushaltskontrolle. Hierauf konzentriert sich der intergouvernementale Fiskalpakt mit der Einführung einer Schuldenbremse im nationalen Verfassungsrecht und umgekehrten Mehrheitsentscheidungen im Aufsichtsverfahren. Wiederum gilt jedoch, dass der völkerrechtliche Fiskalpakt nur ein Element zur Krisenbewältigung sein soll. Daneben fordern die Staats- und Regierungschefs ausdrücklich eine „zügige Prüfung" der Kommissionsvorschläge vom 23. November 2011 zur Stärkung der haushaltspolitischen Überwachung.

Damit wird ein wichtiger Reformschritt kein Bestandteil des intergouvernementalen Fiskalpakts sein, sondern von den EU-Organen auf Grundlage des geltenden EU-Rechts verwirklicht werden. Hierbei erfolgt auch eine Annäherung an das deutsche Ziel einer größeren Kontrolle der Defizitstaaten durch die EU-Kommission in Form einer verstärkten Überwachung der nationale Haushaltspläne, die nach dem Kommissionsvorschlag zugleich den Stabilitätspakt mit der EFSF/ESM-Aufsicht verbinden und nur knapp unterhalb der Schwelle eines formalrechtlichen Durchgriffsrechts verbleiben soll. All diese Reformschritte sind nach Einschätzung der EUKommission ohne Vertragsänderung möglich.

Rechtsgrundlage dieser Neuerungen ist erneut kein völkerrechtlicher Vertrag, sondern die Neufassung des Art. 136 AEUV, der ein Reformprojekt des gescheiterten Verfassungsvertrags in den Vertrag von Lissabon überführt. Von Anfang an bezweckte die Bestimmung eine Vertiefung der Währungsunion, die nun verwirklicht wird. Erst im September 2011 wurde aufgrund der Norm eine verschärfte Kontrolle der nationalen Haushalts- und Wirtschaftspolitik („Six Pack“) vereinbart, die Fritz Scharpf zu Unrecht als unzulässigen Eingriff in die nationale Politikhoheit geißelt. Auch die neuen Vorschläge mögen die kompetentiellen Grenzen des Art. 136 AEUV austesten. Über ihren Erfolg entscheidet jedoch nicht der intergouvernementale Fiskalpakt, sondern das EUGesetzgebungsverfahren. 


\section{Zulässigkeit völkerrechtlicher Satellitenverträge}

Angesichts der begrenzten Reichweite des intergouvernementalen Fiskalpakts stellt sich die Streitfrage nach dessen Vereinbarkeit mit dem EU-Primärrecht nur in abgeschwächter Form. Es geht um punktuelle Vertragsergänzungen durch einen Komplementärvertrag, der wie ein Satellit die supranationale EU-Integration als Gravitationszentrum überstaatlicher Zusammenarbeit umkreist. Als „Bypass“ begegnet dieser Vertrag weniger gravierenden Einwendungen als eine außervertragliche, intergouvernementale Neugründung einer Wirtschaftsregierung, die einem „Herzinfarkt“ für die supranationale Integrationsmethode gleichkäme.

Dies bedeutet freilich nicht, dass der Fiskalpakt generell zulässig wäre. Vielmehr muss im Einzelfall geprüft werden, ob der Fiskalpakt und die materiellen Bestimmungen des EU-Rechts eingehalten wurden. Dies folgt aus den allgemeinen Regeln des EU-Rechts für "ungleichzeitige" völkerrechtliche Verträge einiger Mitgliedstaaten. Der EuGH verpflichtet die Mitgliedstaaten entgegen dem Völkervertragsrecht auf die Beachtung früheren Unionsrechts beim Abschluss späterer völkerrechtlicher Verträge. Hiernach dürfte der Fiskalpakt gerade aufgrund seiner begrenzten Reichweite weitgehend mit dem EU-Recht vereinbar sein (der Verstoß der umgekehrten Mehrheitsentscheidungen gegen Unionsrecht zeigt nur dann rechtspraktische Wirkungen, wenn ein Mitgliedstaat sich auf die Unvereinbarkeit aktiv beruft). Dies zeigen zwei Überlegungen:

Zum einen konzipiert der EU-Vertrag das 3-\%-Schuldenkriterium als Mindestschwelle, die durch völkerrechtliche Schuldenbremsen verstärkt werden kann (auch wenn eine Umsetzung ohne Vertragsänderung gemäß Art. 123 Abs. 14 AEUV möglich gewesen wäre). Hierbei darf sogar der EuGH eingeschaltet werden, weil Art. 273 AEUV völkerrechtliche Schiedsklauseln ausdrücklich gestattet. Ob neue Befugnisse der Kommission aufgrund des Fiskalpakts der Zustimmung aller Mitgliedstaaten bedürfen, ist nicht abschließend geklärt. Hierauf dürfte es im Ergebnis jedoch nicht ankommen, soweit die Schuldenbremse einzig im Rahmen bestehender Überwachungsverfahren beurteilt wird. Eben diese ermächtigen die Kommission bereits zur Aufsicht über nationale Sparbemühungen.

\section{Fazit}

Oft wurde in den vergangenen Monaten das Ende der supranationalen Integrationsmethode herauf beschworen - und das britische Veto gegen die Vertragsänderung hinterlässt in der Tat den Eindruck, dass die Währungsunion einzig auf intergouvernementaler Grundlage gerettet werden kann. Dies trifft insoweit zu, als die EU-Kommission heute (ebenso wie früher) nicht dem Leitbild einer Bundesstaatsregierung entspricht. Die zentralen politischen Weichenstellungen treffen die Staats- und Regierungschefs. Allerdings scheint speziell das deutsch-französische Duo zuletzt der Illusion einer Allmacht zu erliegen. Anders kann man das Insistieren auf einer Vertragsänderung kaum erklären, die nur partiell über die Gestaltungsoptionen des geltenden Rechts hinausreicht.

Wenn die Umsetzung der jüngsten Reformbeschlüsse nur teilweise dem Völkerrecht unterfällt, belegt dies die Leistungsfähigkeit der supranationalen Gemeinschaftsmethode. Diese garantiert, dass die politischen Vorgaben der Staats- und Regierungschefs im Interesse aller, auch der kleinen, Mitgliedstaaten umgesetzt werden. Der Fiskalpakt bestätigt den Eindruck einer rechtlich-institutionellen Kehrtwende nicht. Hieraus sollte auch die juristische Diskussion in Deutschland ihre Konsequenzen ziehen, deren Binnendiskurs sich auf die Rolle des Grundgesetzes, der Bundesregierung, des Bundestags und des BVerfG konzentriert. Dies ist eine Verkürzung der Debatte. Europa kann rechtlich und institutionell nicht allein in nationalstaatlichen Kategorien verstanden werden.

Daniel Thym ist Inhaber eines Lehrstuhls für Öffentliches Recht, Europarecht und Völkerrecht an der Universität Konstanz. 